\title{
Just the facts: initiating mechanical ventilation in the emergency department
}

\author{
George Mastoras $^{1}\left[\right.$ D Sara H. Gray ${ }^{2}$
}

Received: 16 March 2021 / Accepted: 8 June 2021 / Published online: 21 June 2021

() The Author(s), under exclusive licence to Canadian Association of Emergency Physicians (CAEP)/ Association Canadienne de Médecine d'Urgence (ACMU) 2021

\section{Clinical scenario}

A 58-year-old man presents for fever, cough, and respiratory distress. His $\mathrm{SpO} 2$ is $85 \%$ on $15 \mathrm{~L}$ of oxygen and you note altered mental status. Chest X-ray shows multi-lobar pneumonia. You decide to intubate for work of breathing and expected clinical course. While preparing for airway management, you wonder to yourself "how will I optimize him on the ventilator?".

\section{Key questions}

\section{What basic principles guide the selection of a mechanical ventilation strategy?}

Ultimately, while ventilators have many nuanced parameters and outputs of interest, a few basic principles can help guide the initial approach. When a patient requires mechanical ventilation, there are three over-arching objectives that help structure the conceptualization [1]:

a. Ventilation: the process of exchanging air in alveoli. Typically, ventilated patients receive active (positivepressure) inhalation followed by passive exhalation. The efficacy of ventilation is reflected in the partial pressure of carbon dioxide ( $\mathrm{pCO} 2$ ) on a blood gas.

George Mastoras

george.mastoras@gmail.com

1 Division of Emergency Medicine, Department of Medicine, UHN Toronto General Hospital, University of Toronto, R Fraser Elliott Building, Ground Floor Room 480, 200 Elizabeth Street, Toronto, ON M5G 2C4, Canada

2 Divisions of Emergency Medicine and Critical Care, Department of Medicine, University of Toronto, Unity Health, Toronto, ON, Canada b. Oxygenation: the diffusion of oxygen from alveoli into the bloodstream. This is reflected in the oxygen saturation $(\mathrm{SpO} 2)$ and partial pressure of oxygen $(\mathrm{PaO} 2)$ on arterial blood gas.

c. Patient synchrony: the selection of a ventilator "mode" determines the degree of patient comfort during mechanical ventilation. Different modes are also correlated with the risk of complications and ICU length of stay.

\section{How do I optimize ventilation?}

Ventilation is achieved by delivering an amount of inhaled air (tidal volume, $V_{T}$ ) several times per minute (Respiratory Rate). The product of these two parameters is the minute ventilation $\left(\mathrm{MV}=V_{T} \times \mathrm{RR}\right)$, typically ranging from 4-8 L/ min in healthy adults, though in many critically ill patients can rise considerably due to higher metabolic demands (e.g., sepsis, poisonings).

Delivery of a tidal volume can be achieved in one of two ways:

a. a fixed volume of breath is delivered at a set rate of gas flow (inspiratory flow rate), called volume-control; or,

b. an inspiratory pressure $\left(P_{\text {insp }}\right)$ is applied to the airways for a set period of time (i-time), called pressure-control.

Either strategy aims to achieve the same result: sufficient $V_{T}$ to maintain the targeted $\mathrm{pCO} 2$ for the clinical condition.

"Lung protective" ventilation is associated with improved morbidity and mortality [2], and targets a relatively low $V_{T}$ of $6 \mathrm{~mL} / \mathrm{kg}$ of ideal body weight per breath $(4-8 \mathrm{~mL} / \mathrm{kg})$ and plateau pressure $\left(P_{\text {plat }}\right)$ - the measure of peak pressure applied at the small airways-maintained below $30 \mathrm{cmH} 2 \mathrm{O}$. Higher $V_{T}$ should be avoided to prevent lung over-distention. Most patients will require 12-18 breaths/minute to achieve an adequate MV. Serial pCO2 can be measured to reflect whether the MV achieved is adequate-if the $\mathrm{pCO} 2$ remains above the goal, increasing MV will reduce it. 


\section{How do I optimize oxygenation?}

The diffusion of oxygen across the alveolar-capillary membrane can be influenced by factors such as interstitial edema or fibrosis, intrapulmonary shunting, and the oxygen gradient determined by the $\mathrm{FiO} 2$. In critically ill patients, obstacles to effective diffusion are often present and higher $\mathrm{FiO} 2$ is required.

Positive end-expiratory pressure (PEEP) also plays a central role in supporting oxygenation. PEEP improves diffusion primarily through recruitment of collapsed alveoli, resulting in a larger surface area over which diffusion can occur. The classic ARDSnet study showed that in patients with hypoxic respiratory failure, the up-titration of PEEP helps reduce the overall $\mathrm{FiO} 2$ required to maintain oxygenation, and is associated with improved survival [3].

$\mathrm{FiO} 2$ and PEEP should be carefully increased in hypoxic patients and titrated according to the $\mathrm{SpO} 2$ or $\mathrm{PaO} 2$. Ideally, $\mathrm{FiO} 2$ should be maintained at the lowest amount required to reach the oxygenation target to avoid hyperoxia, though the precise amount required will vary with patient physiology.

\section{How do I achieve patient comfort and synchrony?}

Patient comfort is driven by several factors including adequate sedation and analgesia [4] and mode of ventilation. Three fundamental modes exist, though modern ventilators typically offer a blend of these:

a. Assist:Control (A:C): also known as Pressure- or Volume-Control; a "simple ventilator" which delivers $V_{T}$ in a time-cycled fashion, typically for apneic, sedated, or paralyzed patients. Patient-triggered breaths can either be ignored or delivered as an additional "full" programmed breath. A:C modes are associated with longer times to extubation compared with spontaneous breathing.

b. Intermittent minute ventilation (IMV) attempts to "synchronize" scheduled breaths to the patient's respiratory efforts and cadence, aiming to achieve the MV. When extra breaths are requested, the patient draws air spontaneously; this can be "assisted" using Pressure Support. IMV may lead to earlier extubation versus $\mathrm{A}: \mathrm{C}$, but in patients with underlying lung pathology or respiratory muscle fatigue, it may prolong weaning [5]. c. Spontaneous Breathing modes: the patient must initiate every breath. No $V_{T}$ or rate is programmed and minute ventilation is patient-determined. Pressure Support, a form of $P_{\text {insp, }}$, is typically applied to ease work of breathing whenever and for however long a breath is drawn. PEEP is usually applied at end-exhalation. These modes are associated with the least respiratory deconditioning and support earlier extubation but require a patient who is breathing spontaneously and capable of sufficient work of breathing.

\section{What is the difference between a pressure- vs. volume-control strategy? Which is superior?}

Debates surrounding the most appropriate strategy for different clinical conditions (e.g., COPD exacerbation, severe ARDS) often miss that these choices are inextricably linked. In volume-controlled ventilation, a fixed volume of gas $\left(V_{T}\right)$ is delivered at a set flow, so the $P_{\text {insp }}$ will fluctuate based on lung compliance and synchrony. In pressure-controlled ventilation, $P_{\text {insp }}$ is fixed, so the amount of gas delivered in each breath may accordingly vary, under- or over-achieving the desired $V_{T}$.

Attention is better focused on the outcomes of whichever strategy was selected. If volume-control is used, careful attention must be given to the inspiratory and plateau pressures to ensure the avoidance of lung injury. When pressure-control is chosen, clinicians must remain vigilant that the desired minute ventilation is being achieved, and adjust either $P_{\text {insp }}$ or RR to accommodate.

\section{Case resolution}

Following intubation you confer with your respiratory therapist. Given the increased metabolic demand from fever you target an initial MV of $10 \mathrm{~L} / \mathrm{min}$. To manage hypoxia you advocate for titrating PEEP alongside $\mathrm{FiO} 2$ and plan a lungprotective $V_{T}$ based on ideal body weight. With the patient still paralyzed you elect to start volume-control (A:C), with a plan to pivot towards a spontaneous breathing mode when the paralytic wears off.

Your initial ventilator settings (Fig. 1) for this $80 \mathrm{~kg}$ man are: $V_{\mathrm{T}} 6 \mathrm{~mL} / \mathrm{kg}$, RR 20, PEEP 12, FiO2 50\%. The patient appears comfortable on these settings, with stable vitals and a Sp02 94\%. You note the I:E of 1:5 and a safe $P_{\text {plat }}$ of $22 \mathrm{cmH} 20$. Satisfied with your ventilation strategy, you consult ICU. 
Fig. 1 Schematic to help understand the geography of a typical ventilator display. Note the parameters highlighted across the bottom and top-left of the screen: these correspond to the inputs outlined in the article

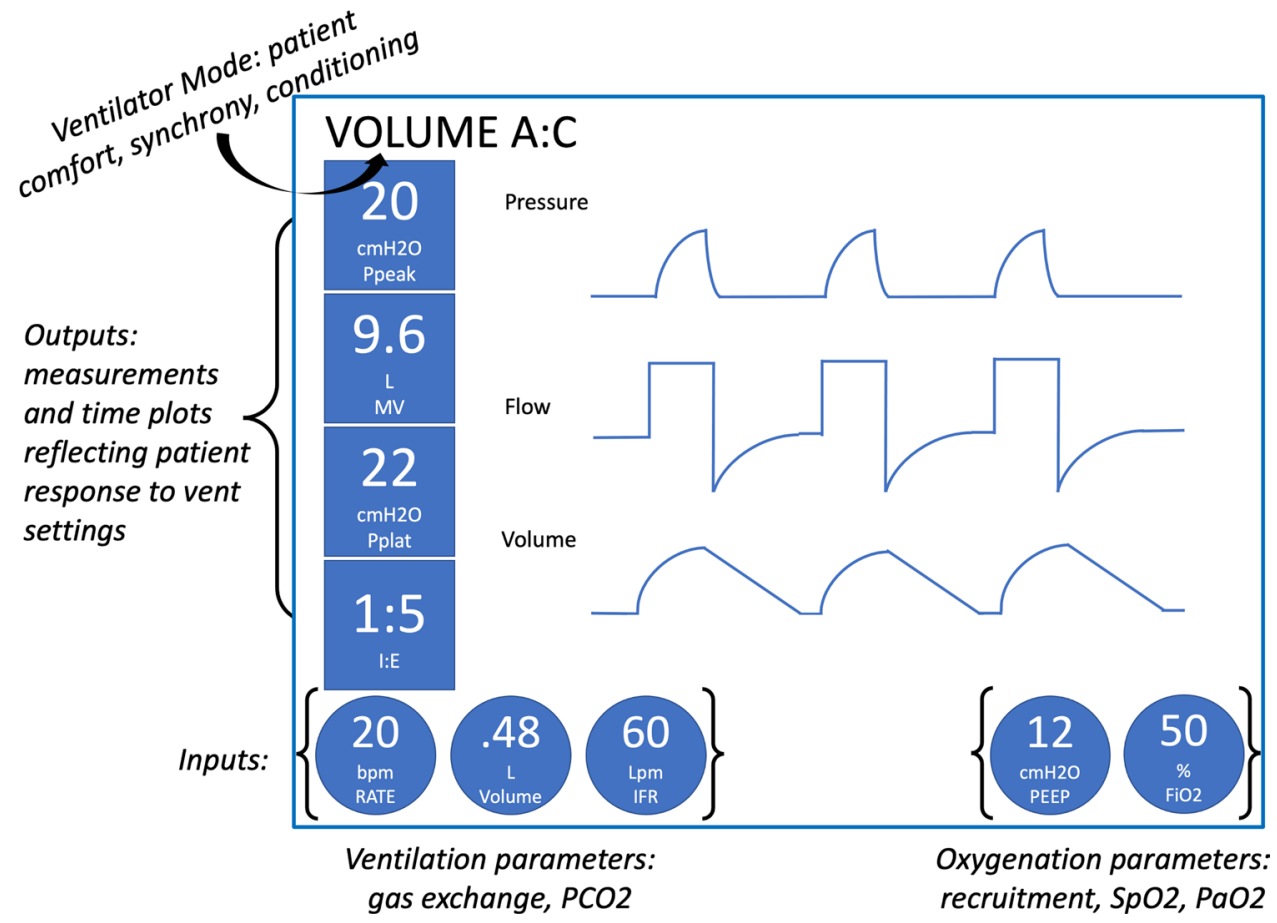

\section{Declaration}

Conflict of interest No conflicts to declare.

\section{References}

1. Acho M, Lee AC, Burton W. Ventilators for nonintensivists. Basic ventilator parameters. ATS Sch. 2020. https://doi.org/10.34197/ ats-scholar.2020-0041VO.

2. Fan E, Brodie D, Slutsky AS. Acute respiratory distress syndrome: advances in diagnosis and treatment. JAMA. 2018;319(7):698710. https://doi.org/10.1001/jama.2017.21907.
3. The Acute Respiratory Distress Syndrome Network. Ventilation with lower tidal volumes as compared with traditional tidal volumes for acute lung injury and the acute respiratory distress syndrome. N Engl J Med. 2000;342:1301-8. https://doi.org/10.1056/ NEJM200005043421801.

4. Lembersky O, Golz D, Kramer C, Fantegrossi A, Carlson JN, Walls RM, Brown CA 3rd, NEAR Investigators. Factors associated with post-intubation sedation after emergency department intubation: a report from The National Emergency Airway Registry. Am J Emerg Med. 2020;38(3):466-70. https://doi.org/10. 1016/j.ajem.2019.05.010.

5. Kacmarek RM, Branson RD. Should intermittent mandatory ventilation be abolished? Respir Care. 2016;61(6):854-66. https://doi. org/10.4187/respcare.04887. 